\title{
Exploit and Repurpose? Targeting the Complex Rules of GADD
}

\section{Jamie Burke and Deepak Bhatia*}

Department of Pharmaceutical Sciences, College of Pharmacy, Northeast Ohio Medical University, Rootstown, OH 44272, USA

Cancer is a worldwide health epidemic. Even with mounting volumes of molecular revelations, it remains elusive, incredibly complex and persistently fatal. The inherent plasticity or stem cell like property of a cancer cell is one aspect that makes understanding the molecular etiology of cancer so complex. It's also the reason why conventional treatments of chemotherapy, radiation and surgery fail and why developing alternative therapies targeting the network of cellular regulation remain an area of intense research. Recent whole genome scans in breast cancer and lung cancer have identified mutations in genes of specific tumor subtypes with the hope of finding potential targets that would respond to drugs currently available or in clinical trials. Some success has already been seen from drugs designed based on mutational analysis as in the development of Gleevec for chronic myelogenous leukemia and Herceptin in breast cancer. Unfortunately, because genetic profiles of tumors are so heterogenous, a resulting treatment may only be effective for a small population as seen with Herceptin limited to a Her-2 genetic profile. Although Gleevec is a tremendous improvement and clearly a success for prolonging life by lengthening the time before relapse, resistance develops as it is unable to touch the population of cancer stem-cells in the quiescent, G0 phase. At this point, it's still not clear how cancer stem cells arise or if a cancer stem cell phenotype is reversible but in several types of normal stem cells, reducing stress signals through Cyclin-dependent kinase (CDK) inhibitors and FOXO transcription factors are thought to maintain quiescence and facilitate long-term maintenance. Since cancer cells have the ability to develop drug resistance from genotoxic stress and evade therapy while in a quiescent state, it makes sense to examine genes and gene products in cancer cells that are major players in stress mediated growth regulation and DNA repair. Specifically, the Growth Arrest and DNA damage (GADD)-inducible gene family and protein members are important candidates for further investigation.

The GADD family of proteins including, GADD34, GADD45a, GADD45 $\beta$, GADD45 $\gamma$, and GADD153 have all been shown to be up regulated by a variety of cellular stresses. It was found that GADD $45 a$ had differential activation based on the type of genotoxic stress. While ionizing radiation evoked the canonical tumor suppressor p53 leading to direct downstream activation of GADD45a, other insults like UV radiation triggered products of MAP kinase pathways that activated GADD45 $\alpha$ independent of p53. Additional functional assays determined that GADD45a binds proteins important for nucleotide excision repair, cell cycle arrest and apoptosis and its expression profile in most often down regulated in a variety of cancers [1]. Investigations on the epigenetic profiles of GADD45 family genes showed distinction between transcripts based on tumor type but similar genomic deregulation by CpG promoter methylation [1-3]. Identifying promoter suppression and gene deregulation has become a common practice in molecular cancer research and more recently, inclusive of genomic scans and tumor profiling. Although the process of discovering epigenetic modulation is complex in both procedure and mechanics, once begotten, interpreting the results is often confined to a priori, of gene to protein, and assumed causality. These areas may be ideal targets for demethylating drugs but as it turns out, finding aberrantly methylated genes in cancer is not a straight shot to a simple answer.

Recent data on GADD protein dynamics indicate a need for defining a complex set of rules for epigenetic regulation. Some studies have shown that the GADD45a protein promotes DNA demethylation in a repair-mediated process and purport "DNA repair is in fact the heart of epigenetic gene-activation [4]. The initial findings that GADD45a could demethylate were challenged by others, reassessed by the claimant and spurred others to join and probe further [5]. More recent studies found that GADD45a bound to hemimethylated DNA and recruited Dnmt1 is supportive of some role in epigenetic modulation [6]. To understand if GADD45a protein is capable of epigenetic modification will no doubt require more investigation. Other protein interactions of GADD45a include RNA binding, as demonstrated by co-localization with RNA helicase p68 in nuclear speckles. The propensity for GADD proteins to function as an RNA binding protein or recruit DNMT1 and alter chromatin structure or other binding promiscuity in response to genotoxic stress may have arose from years of selective pressure. GADD45a, $\beta$ and $\gamma$ proteins also share homology with a ribosomal protein domain but it needs to be assessed what role they play in RNA binding, either directly or in concerted effort and what independent roles each isoform may have specific to cellular duress. Furthermore, post-transcriptional events like mRNA maintenance through positive feedback of post-translational mechanisms exemplify GADD duping the system. Interestingly, Reinhardt and colleagues (7) found that even without p53 function, GADD45a has an alternative route for DNA repair through the MAP kinase, $\mathrm{p} 38 / \mathrm{MK}$ pathway. It appears that a late G2/M checkpoint and cell arrest that might otherwise be initiated by 553 is maintained by keeping active levels of MK-2 in the cytoplasm to act as an inhibitor of downstream CyclinB/Cdk. It's thought that RNA binding proteins on GADD45a mRNA have independent associations with both p38 and MK-2 and through phosphorylation, drive translation of GADD $45 a$ mRNA and increase protein levels. In the cytoplasm, GADD45a protein interacts with p38 and increases cytoplasmic levels of active MK-2. In this way, transcriptional inhibition, essentially gene expression that maintains cell cycle arrest appears to be driven by GADD45 $\alpha$ through positive stimulation of GADD 45 $a$ mRNA and protein [7]. The molecular kinetics of GADD proteins specific to the type of cancer and type of genotoxic stress in relation to the timing of cell cycle checkpoint genes needs further investigation. Others have reported that GADD45a protein physically interacts with $\beta$-catenin and Caveolin- 1 in HeLa and MEF cells and impacts the localization of $\beta$-catenin to the cell membrane rather than the nucleus [8]. Again, in this scenario, GADD45a protein has a driving role in transcriptional inhibition. Additionally in MEF cells, GADD45a was found to regulate and alter gene expression specifically related to cell communication and focal adhesion pathways but not at

*Corresponding author: Deepak Bhatia, Department of Pharmaceutical Sciences, College of Pharmacy, Northeast Ohio Medical University, Rootstown, $\mathrm{OH} 44272$ USA, Tel: 330-325-6482; Fax: 330-325-5936; E-mail: dbhatia@neomed.edu

Received November 20, 2012; Accepted November 21, 2012; Published November 23, 2012

Citation: Burke J, Bhatia D (2012) Exploit and Repurpose? Targeting the Complex Rules of GADD. Clin Exp Pharmacol S5:e001. doi:10.4172/2161-1459.S5-e001

Copyright: ( 2012 Burke J, et al. This is an open-access article distributed under the terms of the Creative Commons Attribution License, which permits unrestricted use, distribution, and reproduction in any medium, provided the original author and source are credited. 
Citation: Burke J, Bhatia D (2012) Exploit and Repurpose? Targeting the Complex Rules of GADD. Clin Exp Pharmacol S5:e001. doi:10.4172/21611459.S5-e001

the gene level through epigenetic modifications or otherwise, rather enigmatically from "aberrant modulation" of GADD45a [9]. These studies suggest that GADD is a major player in cancer and that normal function of protein and RNA interactions are extremely important to gene expression. It also suggests a highly evolved mechanism that may, in some cancer cells, still get the job done, but perhaps in other cancer cells, may have been breached. Functional roles of GADD protein in DNA repair, cell cycle control, and suppressing metastatic potential have all been very recently demonstrated. Add RNA maintenance and regulation and we have a better picture for assigning rules to genomic deregulation in a tumor profile. The mechanisms that GADD respond to and regulate are relevant to the development of drug resistance in cancer. The promiscuous protein binding of GADD, the potential for epigenetic modulation by GADD, and the spatial and temporal location of GADD are all potential candidates for targeted therapy. Could we exploit or re-purpose these modulations? And if so, is GADD modulation really the heart of a cancer cell's plasticity or will a metastatic advantage arise elsewhere and dupe our system? The recent efforts to decode the genomic landscape and perhaps find suitable targets relevant to currently available drugs or drugs in clinical trials may be the best approach thus far. However, cancer genome scans reveal no less of a chaotic mess and choosing the right drug for that landscape involves a confounding amount of trial and error. There is a pressing need for continued functional, kinetic and contextual assays of proteins and RNA products of genes like GADD that are involved in the inhibition of a cancer stem cell. These studies may ultimately provide us the rules to specifically target the stemness of cancer cells.
In the trend toward molecularly targeted medicine, treatment based on a tumor profile will be greatly enhanced by adjunct therapy targeting these novel mechanisms.

\section{References}

1. Cretu A, Sha X, Tront J, Hoffman B, Liebermann DA (2009) Stress sensor Gadd45 genes as therapeutic targets in cancer, Cancer Ther 7: 268-276.

2. Zerbini LF, Libermann TA (2005) GADD45 deregulation in cancer: frequently methylated tumor suppressors and potential therapeutic targets. Clin Cancer Res 11: 6409-6413.

3. Zumbrun SD, Hoffman B, Liebermann DA (2009) Distinct mechanisms are utilized to induce stress sensor gadd $45 \mathrm{~b}$ by different stress stimuli. J Cell Biochem 108: 1220-1231.

4. Niehrs C, Schäfer A (2012) Active DNA demethylation by Gadd45 and DNA repair. Trends Cell Biol 22: 220-227.

5. Jin SG, Guo C, Pfeifer GP (2008) GADD45A does not promote DNA demethylation. PLoS Genet 4: e1000013.

6. Lee B, Morano A, Porcellini A, Muller MT (2012) GADD45ît inhibition of DNMT1 dependent DNA methylation during homology directed DNA repair Nucleic Acids Res 40: 2481-2493

7. Reinhardt HC, Cannell IG, Morandell S, Yaffe MB (2011) Is post-transcriptional stabilization, splicing and translation of selective mRNAs a key to the DNA damage response? Cell Cycle 10: 23-27.

8. Ji J, Liu R, Tong T, Song Y, Jin S, et al. (2007) Gadd45a regulates beta-catenin distribution and maintains cell-cell adhesion/contact. Oncogene 26: 6396-6405.

9. Shan Z, Li G, Zhan Q, Li D (2012) Gadd45a inhibits cell migration and invasion by altering the global RNA expression. Cancer Biol Ther 13: 1112-1122. 